Methods: Data are taken from an epidemiological survey conducted with a national clustered sample of 3998 Australian adults. Following the presentation of a vignette describing depression or schizophrenia, respondents were asked a series of questions relating to their knowledge and recognition of the disorder, beliefs about the helpfulness and harmfulness of helping professionals and treatments, likely outcomes and causes, and personal and perceived stigma.

Results: Participant age was coded into five categories and cross-tabulated with mental health literacy variables. Multiple comparisons between the youngest age group (18-24) and all other groups showed that although young adults were better than those aged $70+$ at correctly recognizing depression and schizophrenia, they were more likely to misidentify schizophrenia as depression. For those who received the depression vignette, younger adults differed from older age groups in terms of their beliefs about the helpfulness and harmfulness of certain treatments, and personal stigma. Differences were also observed between younger and older adults who received the schizophrenia vignette, specifically for helpfulness and harmfulness ratings, and beliefs about causes.

Conclusions: Differences in mental health literacy across the adult life span suggest that more specific, age-appropriate messages about mental health are required to inform different age groups. The tendency for young adults to 'overidentify' depression perhaps signals the need for awareness campaigns to focus on differentiation between mental disorders.

\section{A randomized double-blind trial of right prefrontal cortex low-frequency transcranial magnetic stimulation in major depression}

\section{P Fitzgerald', ZJ Daskalakis², S Huntsman³, R Gunewardene ${ }^{4}$, J Kulkarni'}

'Alfred Psychiatry Research Centre and Monash University Department of Psychological Medicine; ${ }^{2}$ Centre for Addiction and Mental Health, Toronto, Ontario, Canada; ${ }^{3 P a l m}$ Beach Currumbin Clinic, Gold Coast, Queensland, Australia; and ${ }^{4}$ Mosman Private Hospital, Mosman, New South Wales, Australia

Background: Low-frequency right prefrontal repetitive transcranial magnetic stimulation (rTMS) appears to have antidepressant properties although the effectiveness of this treatment in clinical practice has not been assessed and the optimal stimulation parameters defined. The boundaries of 'low-frequency' stimulation are not clear.

Methods: A total of 122 patients with treatmentresistant depression were randomized to either 1- or $2-\mathrm{Hz}$ stimulation over right prefrontal cortex (PFC) (one single 15-min train) for 2 weeks. A second 2 -week period of treatment was provided for patients showing initial response ( $>20 \%$ reduction in HAMD score).

Results: One hundred eighteen patients received a full 2 weeks of treatment $(63-1 \mathrm{~Hz}, 55-2 \mathrm{~Hz})$. There was a mean reduction in HAMD scores of 30.1 $\pm 29.8 \%$ in the $1-\mathrm{Hz}$ group and $33.2 \pm 31.7 \%$ in the $2-\mathrm{Hz}$ group $(P>0.05)$. Seventy-eight patients received a further 2 weeks of treatment. Over the full 4 weeks, there was a reduction in HAMD scores of $62.2 \pm 25.1(1-\mathrm{Hz}$ group $)$ and $61.3 \pm 25.2 \%(2-\mathrm{Hz}$ group) $(P>0.05)$. Thirty of $63(48 \%)$ patients in the $1-\mathrm{Hz}$ group and 29 of $55(53 \%)$ patients in $2-\mathrm{Hz}$ group met response criteria $(>50 \%$ reduction HAMD score) at study end. There was no difference in clinical response between groups.

Conclusions: Despite a heterogeneous sample, a significant proportion $(\sim 50 \%)$ of patients met clinical response criteria following treatment. There was no difference in clinical response to $1-$ or $2-\mathrm{Hz}$ rTMS applied to right dorsolateral PFC. This suggests that $2-\mathrm{Hz}$ right $\mathrm{PFC}$ rTMS has antidepressant properties but offers no advantage over $1-\mathrm{Hz}$ stimulation despite a twofold increase in pulse number.

\section{A meta-analytic study of changes in brain activation in depression}

\section{P Fitzgerald ${ }^{1,2}$, J Maller ${ }^{1,2}, \mathrm{~K} \mathrm{Hoy}^{1,2}, \mathrm{~T}$ Oxley ${ }^{1,2}$, Z Daskalakis ${ }^{3}$, A Laird ${ }^{4}$}

${ }^{1}$ Alfred Psychiatry Research Centre, The Alfred; ${ }^{2}$ Monash University Department of Psychological Medicine, Melbourne, Australia; ${ }^{3}$ Centre for Addiction and Mental Health, College Street Site, Toronto, Ontario, Canada; and ${ }^{\text {RResearch Imaging }}$ Centre, The University of Texas Health Science Centre San Antonio, USA

Introduction: A large number of studies with considerably variable methods have been performed to investigate brain regions involved in the pathophysiology of major depressive disorder. The aim of this study was to use a quantitative meta-analytic technique to synthesize the results of much of this research.

Methods: Three separate quantitative meta-analytical studies were conducted using the activation likelihood estimation technique. Analysis was performed of studies conducted at rest comparing brain activation in patients with depression and controls, studies conducted of brain changes associated with antidepressant medication treatment and studies comparing brain activation patterns induced by the induction of positive or negative emotion in patients with depression compared with controls.

Results: The results of the study indicated a complex series of areas of the brain implicated in the pathophysiology depression. This included a network of dorsal regions that are hypoactive in depressed subjects and increase in activity with treatment and a corresponding 